7th International Workshop on Astronomy and

Relativistic Astrophysics (IWARA 2016)

International Journal of Modern Physics: Conference Series

Vol. 45 (2017) 1760061 (4 pages)

(C) The Author(s)

DOI: $10.1142 / \mathrm{S} 2010194517600618$

\title{
Investigating the Prompt Photon Production at the LHC Energies
}

\author{
Glauber S. dos Santos and Magno V. T. Machado \\ Instituto de Física \\ Universidade Federal do Rio Grande do Sul (UFRGS) \\ Av. Bento Gonçalves 9500, 91501-970 Porto Alegre, RS, Brazil \\ gss.fisica@gmail.com,magnus@if.ufrgs.br
}

Published 15 August 2017

\begin{abstract}
We investigate the rapidity and transverse momentum distributions of the prompt photon production at the LHC energies considering the color dipole approach. We compare the predictions from distinct models for the dipole cross section, where parton saturation models at high energies are expected to be important at the forward rapidities in $p p$ and $p A$ collisions at the LHC.
\end{abstract}

Keywords: Prompt Photon, Saturation Effects, Transverse Momentum.

\section{Prompt Photon Production in $p p$ and $p A$ Collisions}

A photon produced in hadronic collision is considered direct when it does not originate from the decay of a hadron, such as $\pi^{0}$ or $\eta$, or when produced with large transverse momentum $p_{T}$. Similar to the DIS, the prompt photon production process can be seen in the target rest system, where the production mechanism resembles a bremsstrahlung ${ }^{1}$. A quark or antiquark from the projectile hadron radiates a real photon while collides with the target. This radiation can occur before or after the quark (antiquark) is scattered by the target. Therefore, we can apply the color dipole formalism to describe the radiation processes ${ }^{2}$. In Ref. ${ }^{1}$, was derived the $p_{T}$ momentum distribution of the transverse photon bremsstrahlung, integrated over the final quark transverse momentum,

$$
\begin{aligned}
\frac{d \sigma(q p \rightarrow q \gamma p)}{d(\ln \alpha) d^{2} \vec{p}_{T}} & =\frac{1}{(2 \pi)^{2}} \sum_{i n, f} \int d^{2} \vec{r}_{1} d^{2} \vec{r}_{2} e^{\left[i \vec{p}_{T} \cdot\left(\vec{r}_{1}-\vec{r}_{2}\right)\right]} \Psi_{T}^{* \gamma q}\left(\alpha \vec{r}_{1}\right) \Psi_{T}^{\gamma q}\left(\alpha \vec{r}_{2}\right) \\
& \times \frac{1}{2}\left[\sigma^{d i p}\left(x, \alpha \vec{r}_{1}\right)+\sigma^{d i p}\left(x, \alpha \vec{r}_{2}\right)-\sigma^{d i p}\left(x, \alpha\left(\vec{r}_{1}-\vec{r}_{2}\right)\right)\right]
\end{aligned}
$$

This is an Open Access article published by World Scientific Publishing Company. It is distributed under the terms of the Creative Commons Attribution 4.0 (CC-BY) License. Further distribution of this work is permitted, provided the original work is properly cited. 
where $\vec{r}_{1}$ and $\vec{r}_{2}$ are the quark-photon transverse separations. The parameter $\alpha$ is the relative fraction of the quark momentum carried by the photon. The energy dependence of the dipole cross section comes via $x_{2}=\left(p_{T} / \sqrt{s}\right) e^{-y}$, where $y$ is rapidity variable of the direct photon. The hadronic cross section can then be obtained due the convolution of the partonic cross section with the proton structure function $F_{2}^{p}$. Therefore, for hadron-hadron collisions, the transverse momentum distribution for production of direct photon is given by ${ }^{3,4}$,

$$
\frac{d \sigma(p p \rightarrow \gamma X)}{d y d^{2} \vec{p}_{T}}=\int_{x}^{1} \frac{d \alpha}{\alpha} F_{2}^{p}\left(\frac{x}{\alpha}, \mu^{2}\right) \frac{d \sigma(q p \rightarrow q \gamma p)}{d(\ln \alpha) d^{2} \vec{p}_{T}}
$$

Furthermore, we need to identify the hard scale $\mu$ entering in the proton structure function, $\mu^{2}=p_{T}^{2}$, and the energy scale of the dipole cross section, $x=x_{2}$. For $F_{2}^{p}$ we have taken the parametrization given in Ref. ${ }^{5}$, which is valid in the kinematic range we are interested in. The color dipole formalism provides a simple generalization of the description of the prompt photon production in $p p$ to $p A$ collisions. The nuclear extension of the Eq. (2) is made by replacing $\sigma^{\text {dip }}$ for its corresponding nuclear version,

$$
\sigma_{A}^{d i p}(x, \alpha \vec{r})=2 \int d^{2} b\left\{1-\exp \left[-\frac{1}{2} \sigma^{d i p}(x, \alpha \vec{r}) T_{A}(b)\right]\right\}
$$

where $T_{A}$ stand for the nuclear profile function. Then for the nuclear scenario, the transverse momentum distribution is written as, ${ }^{1}$

$$
\frac{d \sigma(p A \rightarrow \gamma X)}{d y d^{2} \vec{p}_{T}}=\int_{x}^{1} \frac{d \alpha}{\alpha} F_{2}^{p}\left(\frac{x}{\alpha}, \mu^{2}\right) \frac{d \sigma(q A \rightarrow \gamma X)}{d(\ln \alpha) d^{2} \vec{p}_{T}}
$$

Theoretically $\sigma^{d i p}$ is unknown, although there are various parameterizations in the literature. The dipole cross section is modeled according to a scaling variable, $r Q_{s}(x)$, where $Q_{s}(x)$ is the saturation scale. $Q_{s}(x)$ defines the transverse momentum scale where the saturation physics is relevant, namely, the partonic recombination processes take part in scattering at high energies. In general, $Q_{s}(x) \propto x^{-\lambda / 2}$, and grows with energy. According to our proposal for $\sigma^{d i p}$, we will use the GBW ${ }^{6}$ and BUW $^{7}$ analytical parameterizations, which the general form is

$$
\sigma^{d i p}(x, \vec{r} ; \gamma)=\sigma_{0}\left[1-\exp \left(-\frac{r^{2} Q_{s}^{2}}{4}\right)^{\gamma_{\mathrm{eff}}}\right], \quad Q_{s}^{2}(x)=\left(\frac{x_{0}}{x}\right)^{\lambda}
$$

In GBW model, the parameters, fitted from DIS data in HERA including 4 quark flavors are: $\sigma_{0}=29.12 \mathrm{mb}, x_{0}=0.41 \times 10^{-4}$ and $\lambda=0.277$. The parameter of the effective anomalous dimension, $\gamma_{\text {eff }}$, is responsible for producing some differences among the phenomenological models for dipole cross section. In the literature, many models admit: $\gamma_{\text {eff }}=\gamma_{s}+\Delta\left(x, r ; p_{T}\right)$, where $\gamma_{s}$ is the anomalous dimension at 
the saturation scale. The expected behavior for $\Delta\left(x, r ; p_{T}\right)$ is distinct among the parameterizations. While GBW assumes $\gamma_{e f f}=1$, BUW considers that

$$
\gamma_{\mathrm{eff}}=\gamma_{s}+\left(1-\gamma_{s}\right) \frac{\left(\omega^{a}-1\right)}{\left(\omega^{a}-1\right)+b}
$$

where $\omega \equiv p_{T} / Q_{s}$. The parameters $a=2.82$ and $b=162$ are fitted in order to describe the HERA data for the proton structure function. Furthermore, it was shown in Ref. ${ }^{7}$ that the BUW model describes RHIC data on hadro-production, where the values of $x$ probed are considered large. When $\gamma_{e f f}=1$, the BUW model reduces the GBW model, except for the parameters: $\sigma_{0}=21 \mathrm{mb}, x_{0}=3.04 \times 10^{-4}$ and $\lambda=0.288$.

\section{Results}

We call attention that the dipole approach is well suited for high energy processes, i.e., small $x_{2}$, and its range of validity is expected to be near $x_{2}<0.01$. In the following, we shown in Fig. 1 our predictions for direct photon production at the LHC energies, $\sqrt{s}=7$ and $8 \mathrm{TeV}$. The data are extracted from the CMS ${ }^{8}$ and ATLAS ${ }^{9}$ ( $y$ integrated, $0<y \leq 0.6$ ) experiments, respectively. The value achieved of the kinematic region of the dipole formalism, $x_{2}$, in CMS and ATLAS energies equivalent to $p_{T} \sim 70 \mathrm{GeV}$ and $p_{T} \sim 80 \mathrm{GeV}$, correspondingly. In both energies, GBW and BUW models predict slightly different results in $p_{T}<20 \mathrm{GeV}$. Apparently, in the region $20 \mathrm{GeV}<p_{T}<70 \mathrm{GeV}$, the GBW approach improves the description of the data within the error bars. As the $p_{T}$ distribution spectrum increases, $p_{T}>70 \mathrm{GeV}$, the models provide estimates that begin to deviate from the experimental data. However, the description of the experimetal points in CMS is reasonable compared to ATLAS, where the parameterizations give the worst scenario. Now, we investigated the prompt photon production in $p A$ collisions. We calculate the invariant cross section in $p P b$ collisions at the energy of $\sqrt{s}=5.02$ and $8.8 \mathrm{TeV}$ at $y=0$, see Fig. 2. It is expected that saturation effects should be amplified in nuclear reactions, as the nuclear saturation scale, $Q_{s, A}^{2} \sim A^{1 / 3} Q_{s, p}^{2} \sim 7.7 \mathrm{GeV}^{2}$. The following
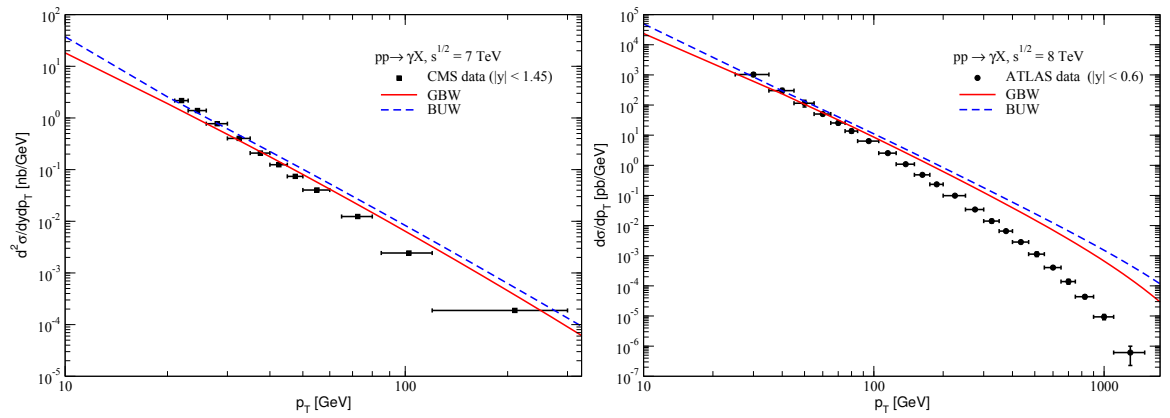

Fig. 1. $p_{T}$ momentum distribution for prompt photon production in $p p$ collisions at the LHC. 

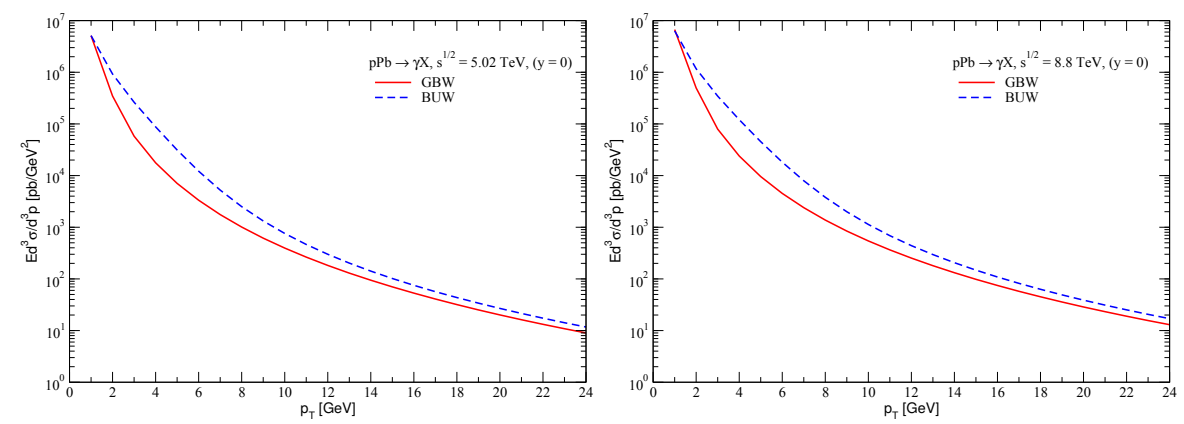

Fig. 2. $p_{T}$ momentum distribution for prompt photon production in $p P b$ collisions at the LHC.

discussion about the estimates is common for different center of mass energies. For $p_{T}<12 \mathrm{GeV}$, GBW and BUW have different results, with the highest deviation being approximately by a factor of $10\left(p_{T}=4 \mathrm{GeV}\right)$. In the direction of large transverse momentum, both parameterizations produce similar predictions. According to our results, we conclude that the saturation physics is not directly relevant for LHC energies at central rapidity, as the saturation scale reaches small values in the range of kinematic data. Taking the GBW model, the maximum value for the saturation scale at the $\mathrm{LHC}$ is $Q_{s, p}^{2} \leq 1.3 \mathrm{GeV}^{2}$, these value is small compared to the transverse momentum, $2 \mathrm{GeV}^{2}<p_{T}^{2}<576 \mathrm{GeV}^{2}$. Therefore, the saturation effects do not play a significant role in the prompt photon production in the experimental range of $p_{T}$.

\section{Conclusions}

In our calculations, the GBW and BUW models tend to provide predictions that describe reasonable the data in large and small transverse momentum, respectively. Moreover, even at the LHC energies, the saturation effects do not take part in the results of the observables. An attempt to change this scenario is to encourage measurements of direct photons in frontal rapidity, as this can be a useful tool for investigating the physics of saturation at small $x$.

\section{References}

1. B. Z. Kopeliovich, A. V. Tarasov and A. Schafer, Phys. Rev. C 59, 1609 (1999).

2. M. V. T. Machado and C. B. Mariotto, Eur. Phys. J. C 61, 871 (2009).

3. B. Z. Kopeliovich, J. Raufeisen and A. V. Tarasov, Phys. Lett. B 503, 91 (2001).

4. B. Z. Kopeliovich et al., Phys. Lett. B 653, 210 (2007).

5. B. Adeva et al., Phys. Rev. D 58, 112001 (1998).

6. K. Golec-Biernat and M. Wüsthoff, Phys. Rev. D 59, 014017 (1999).

7. D. Boer, A. Utermann and E. Wessels, Phys. Rev. D 77, 054014 (2008).

8. CMS Collaboration (V. Khachatryan et al.), Phys. Rev. Lett. 106, 082001 (2011).

9. ATLAS Collaboration (G. Aad et al.), JHEP 1608, 005 (2016). 